\title{
High-grade mutant OmpF induces decreased bacterial survival rate
}

\author{
Zhi-ping Zhao ${ }^{1}$, Ting-ting Liu' ${ }^{1}$, Li Zhang ${ }^{1}$, Min Luo ${ }^{2}$, Xin Nie ${ }^{\circledR}$, Zai-xin Li ${ }^{1}$ and Yu Pan ${ }^{3 凶}$ \\ 1School of Chemistry and Pharmaceutical Engineering, Sichuan University of Science \& Engineering, Zigong, China; 2Journals Department, \\ Chongqing University, Chongqing, China; ${ }^{3}$ College of Horticulture and Landscape Agriculture, Southwest University, Beibei, China
}

\begin{abstract}
OmpF plays very important roles in the influx of antibiotics and bacterial survival in the presence of antibiotics. However, high-grade mutant OmpF and its function in decreasing bacterial survival rate have not been reported to date. In the present study, we cloned a high-grade mutant OmpF (mOmpF) and sequence analysis suggested that over 45 percent of the DNA sequence was significantly mutated, leading to dramatic changes in over 55 percent of the amino acid sequence. mOmpF protein was successfully expressed. When grown in the presence of antibiotic, the bacterial survival rate decreased and the antibiotic inhibition zone became larger with the increase of the mOmpF. It was concluded that concentration of high-grade mutant $\mathrm{mOmpF}$ dramatically influenced the bacterial survival rate. The study presented here may provide insights into better understanding of the relationships between structure and function of OmpF.
\end{abstract}

Key words: antibiotic resistance, $\mathrm{mOmpF}, \mathrm{OmpF}$, outer membrane protein, bacterial survival rate

Received: 18 February, 2014; revised: 11 April, 2014; accepted: 15 April, 2014; available on-line: 13 June, 2014

\section{INTRODUCTION}

Antibiotic resistance has been frequently reported in clinical Gram-negative bacteria responsible for a large number of antibiotic resistant bacterial diseases and has been a serious problem that urgently needs to be addressed (Pages et al., 2008). Antibiotic resistant E. coli strains are considered a classic sample. As for the antibiotic resistance mechanisms, many bacterial strategies have been presented, mainly including limitation of the intracellular access to an antibiotic, production of detoxifying enzymes by enzymatic barrier to degrade or modify the antibiotic and the target protection barrier impairing target recognition and thus the antimicrobial activity (Davin-Regli et al., 2008; Jaffe et al., 1982). It has been reported that antibiotic translocation through the OMP porin of Gram-negative bacteria is the first key step for an antibiotic to act on its specific intracellular targets (Masi \& Pages, 2013). Thus, OMP is the first line of defense of Gram-negative bacteria against external toxic compounds such as antibiotics. Three major OMP porins of very similar sequences and three-dimensional structures have been extensively studied, termed $\mathrm{OmpF}$, OmpC and PhoE (Nikaido, 2003), among which, OmpF and $\mathrm{OmpC}$ are considered to be homologues and exhibit a slight preference for cations, whereas PhoE plays more important roles in the transport of inorganic phosphate and anions (Schulz, 2002; Koebnik et al., 2000).

$\mathrm{OmpF}$ is one of the most important porins and widely found in E. coli. A number of studies on bacterial antibiotic resistance closely correlating with $\mathrm{OmpF}$ porin have been reported. Dupont et al reported that $\mathrm{OmpF}$ is involved in the control of $\beta$-lactams and fluoroquinolones penetration through enterobaccterial outer membrane (Jaffe et al., 1982; Dupont et al., 2007). Yigit et al suggested that repressed expression of OmpF results in imipenem resistance and decreased susceptibility to meropenem and cefepime in Enterobacter aerogenes (Yigit et al., 2002). Cohen et al proposed that reduction in $\mathrm{OmpF}$ levels should be taken into account for the marA-dependent fluoroquinolone resistance (Cohen et al., 1989). OmpF has also been proved, by proteomics technologies, to be related to the transportation of ruthenium complexes through cell membrane and $\mathrm{OmpF}$ porin plays a key role in the transportation of positively charged polypyridyl chlororuthenium complexes into E. coli (Ho et al., 2010). OmpF porin is known to play an important role in the uptake of fluoroquinolone antibiotics by bacteria and binding of fluoroquinolone antibiotic ciprofloxacin to $\mathrm{OmpF}$ in a lipid membrane environment is necessary for such uptake (Fernandes et al., 2007). Moreover, it has been also reported that OmpF plays essential roles in the acid resistance of $E$. coli in the presence of arginine and lysine (Bekhit et al., 2011).

$\mathrm{OmpF}$ is considered to be the primary gateway for antibiotics to enter into the cell (Nikaido, 2003). E. coli $\mathrm{OmpF}$ is a well-defined porin which allows the diffusion of molecules of sizes up to 600 Daltons and thus allows many hydrophilic antibiotics to permeate across the membrane and subsequently enter into cells (Bredin et al., 2003). Structure of OmpF has been well characterized. The E. coli $\mathrm{OmpF}$ is a trimer comprised of 16-stranded anti-parallel $\beta$-barrels (Cowan et al., 1992). It has eight short turns (T1-T8) on the periplasmic side and eight long loops (L1-L8) on the extracellular side connecting the antiparallel $\beta$-strands. L3 loop is particularly long consisting of 33 residues and is a keystone of the channel architecture, constributing to the high conductance and cationic selectivity of $\mathrm{OmpF}$. The structure of $\mathrm{OmpF}$ changes in different conditions. Kefala and coworkers (2010) reported two OmpF structures, one is refined in space group P21 resolved at the resolution of

e-mail: niexinsuse@yahoo.com; pany1020@swu.edu.cn Abbreviations: E. coli, Escherichia coli; OMP, outer membrane protein; LB, Luria-Bertani; IPTG, isopropyl $\beta$-D-1-thiogalactopyranoside; $\mathrm{ORF}$, open reading fragment; $\mathrm{kDa}$, kilo-Dalton.

Accession number: HG738867.1 
$3.8 \AA$ and the other is refined in the P321 space group resolved at the resolution of $4.4 \AA$ (Kefala et al., 2010).

Nevertheless, high-grade mutant $\mathrm{OmpF}$ and its function in decreasing bacterial survival rate have not been reported yet. In the present study, we cloned a highgrade mutant $\mathrm{OmpF}$ from an isolated antibiotic resistant E. coli strain and explored its activities in decrease of bacterial survival rate when grown in the presence of antibiotics. The present study may provide insights into better understanding of the relationships between structure and function of $\mathrm{OmpF}$.

\section{MATERIALS AND METHODS}

Bacterial strains and growth conditions. The multiantibiotic resistant E. coli strain was isolated as described in our previous study (Zhao et al., 2013) and grown at $37^{\circ} \mathrm{C}$ in $\mathrm{LB}$ medium containing $50 \mu \mathrm{g} / \mathrm{mL}$ ampicillin, $30 \mu \mathrm{g} / \mathrm{mL}$ streptomycin. E. coli DH5 $\alpha$ and BL21 (DE3) strains were grown at $37^{\circ} \mathrm{C}$ in $\mathrm{LB}$ medium supplemented with $50 \mu \mathrm{g} / \mathrm{mL}$ kanamycin if necessary.

Cloning and sequencing of mOmpF. Genomic DNA of the isolated multi-antibiotic resistant E. coli strain was purified from $1 \mathrm{~mL}$ of an overnight grown cell culture using the Bacterial DNA Extraction Kit (Omega, USA) according to the manufacturer's instructions. $m O m p F$ forward primer sequence (5'-CGGAATTCATGATGAAGCGCAATAT'TCTGGC-3') and $m \mathrm{OmpF}$ reverse primer sequence (5'-CCCAAGCTTGAACTGGTAAACGATACCCACAGCAAC-3') were designed according to the reported $O m p F$ sequence (Accession No. HG738867.1). EcoRI and HindIII restriction sites were added to the 5'-terminal of $m \mathrm{OmpF}$ forward and reverse primers, respectively. PCR was carried out by using Pfu DNA polymerase (Promega) at $94^{\circ} \mathrm{C}$ for $5 \mathrm{~min}$, followed by 30 cycles of $94^{\circ} \mathrm{C}$ for $30 \mathrm{~s}, 55^{\circ} \mathrm{C}$ for $30 \mathrm{~s}$ and $72^{\circ} \mathrm{C}$ for $2 \mathrm{~min}$. The amplified $m O m p F$ product was purified with Omega DNA Purification Kit and digested with EcoRI and HindIII and subsequently ligated into pET28a cut with the same restriction enzymes, resulting in the $m \mathrm{OmpF}$ expression vector pET28-mOmpF. The expression vector was then sequenced by the Shenzhen Huada Genomics Institute (China) and analyzed with DNAMAN software. After sequencing, the expression vector was transformed into the E. coli BL21 (DE3) strain.

Expression of mOmpF. A $10-\mathrm{mL}$ culture of LB medium supplemented with $50 \mu \mathrm{g} / \mathrm{mL}$ kanamycin was incubated with a single colony of BL21/pET28-mOmpF and grown overnight at $37^{\circ} \mathrm{C}$ with vigorous shaking. In a $100-\mathrm{mL}$ culture of LB medium containing $50 \mu \mathrm{g}$ / $\mathrm{mL}$ kanamycin, $1 \mathrm{~mL}$ of the overnight grown cell culture was inoculated. The culture was incubated for about $2 \mathrm{~h}$ until the $\mathrm{OD}_{600}$ reached $0.6-0.8$ and then induced by the addition of IPTG to a final concentration of 1 $\mathrm{mM} .1 \mathrm{ml}$ of sample was collected at $0 \mathrm{~min}, 20 \mathrm{~min}, 40$ $\min , 1 \mathrm{~h}$ and $2 \mathrm{~h}$, respectively, after induction under the same growth conditions. Induced samples were collected by centrifugation at $7000 \times g$ for $1 \mathrm{~min}$ at $4^{\circ} \mathrm{C}$ and then washed twice with PBS buffer. The treated samples were boiled for $5 \mathrm{~min}$ and then analyzed by SDS/PAGE on a $12 \%$ gel.

Effects of $\mathrm{mOmpF}$ on the survival rate of bacteria. For all the experimental analysis, the expressed cell culture was adjusted to an $\mathrm{OD}_{600}$ of 0.7 and then diluted $2 \times 10^{5}$-fold with LB medium. Then, $50 \mu \mathrm{L}$ of the diluted suspension was plated on the LB agar medium in the presence and absence of kanamycin, respectively. Each experiment was repeated 3 times. The survival rate $R$ was estimated by $R=\frac{N 2}{N 1} \times 100 \%$, in which $N_{1}$ is the number of bacteria that have grown in LB agar medium in the absence of kanamycin and $N_{2}$ is the number of bacteria that have grown in LB agar medium in the presence of kanamycin.

Effects of mOmpF on the inhibition zone. Induced BL21/pET28-mOmpF cell culture was adjusted to an $\mathrm{OD}_{600}$ of 0.4 . LB agar $(1.5 \%)$ medium was first placed in a plate, then $100 \mu \mathrm{L}$ of the cell suspension was added to $100 \mathrm{~mL}$ of the LB agar $(0.7 \%)$ medium and subsequently overlaid on the LB agar $(1.5 \%)$ medium. Wells were prepared by Oxford cup. In each well, 25 $\mu \mathrm{L}$ of $50 \mathrm{mg} / \mathrm{mL}$ kanamycin was added and cultured at $37^{\circ} \mathrm{C}$ for about $8 \mathrm{~h}$. The diameter of the inhibition zone extending laterally around the well was measured by vernier caliper. Each experiment was repeated 3 times.

\section{RESULTS}

\section{Cloning of the mOmpF}

The length of the $m O m p F$ fragment was approximately $1100 \mathrm{bp}$ as expected. However, sequence analysis implied that the length of the ORF of the $m O m p F$ was $903 \mathrm{bp}$, which was dramatically mutated compared with the model $1020 \mathrm{bp} O m p F$. The $m O m p F$ sequence shared only $54.5 \%$ homology with the model OmpF as measured by DNAMAN software. The stop codon "TGA" was unexpectedly located at the $904 \mathrm{bp}$ and thus changed the length of $m \mathrm{OmpF}$ ORF. Consequently, the amino acid sequence of the $\mathrm{mOmpF}$ was also dramatically mutated compared with the model OmpF and it was only $44.6 \%$ identical to that of the model OmpF, as shown in Fig. 1. The model OmpF comprised 340 amino acid residues, whereas the mOmpF contained only 301 amino acid residues. Apparently, the C-terminus of $\mathrm{mOmpF}$ shared very little homology with the model OmpF. The mutant rate was up to $96.6 \%$ from the 283 rd to 340 th amino acid residues at the $\mathrm{C}$-terminus. Furthermore, structure predication of $\mathrm{mOmpF}$ and $\mathrm{OmpF}$ by a Swiss-model indicated that the three-dimensional structure of $\mathrm{mOmpF}$ was hugely altered compared to that of the model E. coli $\mathrm{K}-12$ OmpF (not shown). To further identify the accuracy of sequencing, two other clones were simultaneously sequenced and their sequences turned out to be completely the same.

OmpF porin exists in the form of homotrimers of identical subunits, each consisting of a 16-stranded anti-parallel $\beta$-barrel with eight short turns (T1-T8) and eight long loops (L1-L8). The eight shorts turns and eight long loops are crucial to the structure and function of OmpF. Alterations of the OmpF amino acid residues result in the mutations of the 16 strands, the short turns and the eight long loops. The number of mutant codons from strand 1 to strand 16 was 6 , $3,3,2,1,1,3,8,10,3,7,5,8,10,8$ and 10 , respectively. Consequently, the mutation rate was $37.5 \%$, $21.4 \%, 25 \%, 22.2 \%, 14.3 \%, 12.5 \%, 25 \%, 61.5 \%$, $90.9 \%, 25 \%, 63.6 \%, 38.5 \%, 53.3 \%, 100 \%, 80 \%$ and $100 \%$ for strands 1 to 16 , respectively. It is apparent that the strands were all mutated at different levels. It also follows that the mOmpF comprises only 15 strands because of the alteration. The number of mutant codons was $1,1,2,1,1,0,0$ and 2, for short turns ( $\mathrm{T}$ ) $\mathrm{T} 1$ to $\mathrm{T} 8$, respectively. Thus, the mutation rate was $100 \%, 50 \%, 100 \%, 12.5 \%, 50 \%, 0,0$ and 


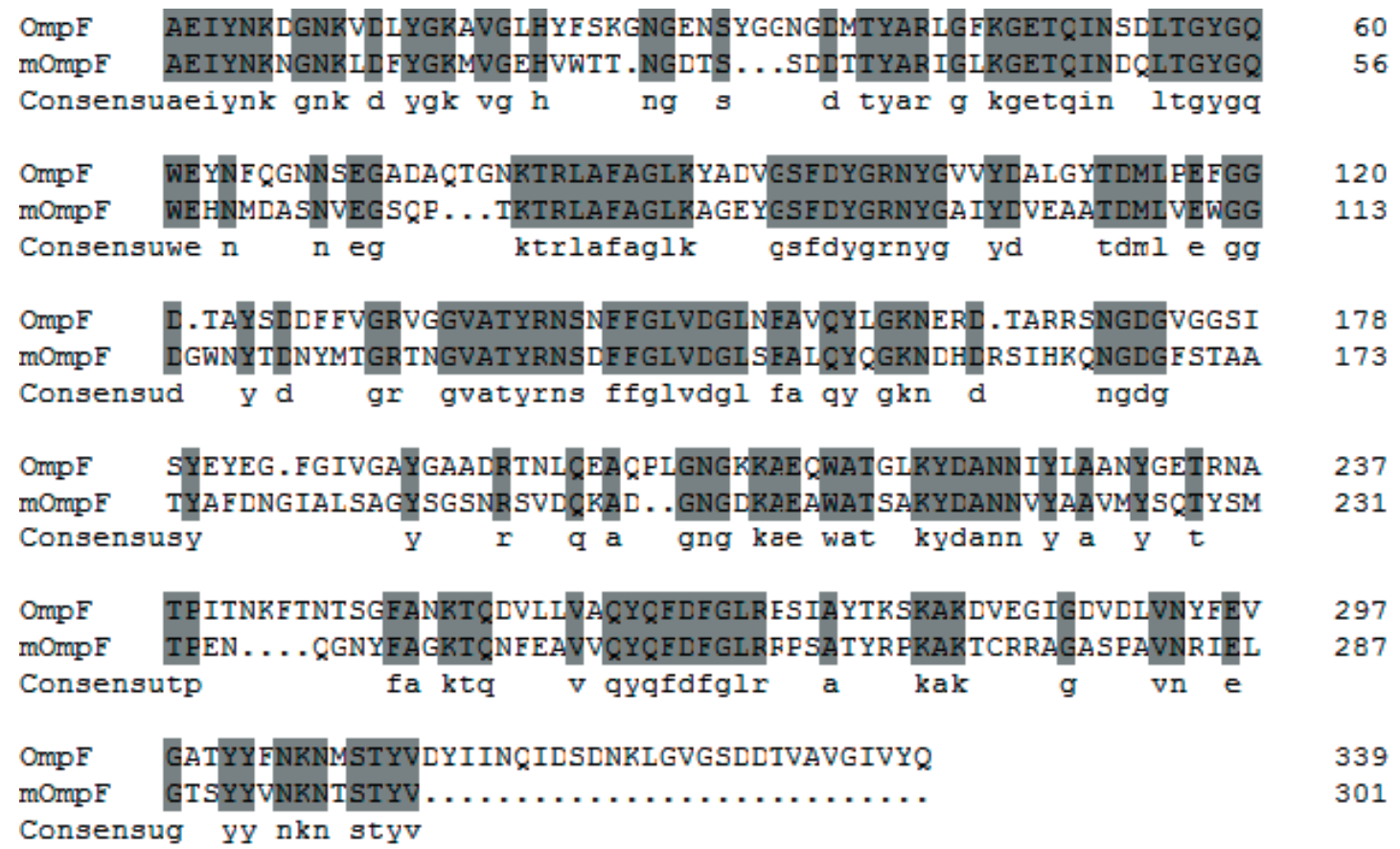

Figure 1. Amino acid sequence alignment of $\mathrm{mOmpF}$ and $\mathrm{OmpF}$.

Table 1. Effects of mOmpF expression on bacterial survival rate

\begin{tabular}{lccc}
\hline \multirow{2}{*}{ Strains } & \multicolumn{3}{c}{ Number of clones } \\
\cline { 2 - 3 } & LB medium with kanamycin & LB medium & \\
\hline BL21/pET28a & 91 & 123 & $73.98 \%$ \\
\hdashline BL21/pET28-mOmpF & 0 & 80 & 0 \\
\hline
\end{tabular}

$100 \%$ for $\mathrm{T} 1$ to $\mathrm{T} 8$, respectively. The number of mutant codons of long loops (L) from L1 to L8 was 10, $11,16,7,8,13,11$ and 15, respectively. And the mutation rate from L1 to L8 was $71.4 \%, 64.7 \%, 48.5 \%$, $87.5 \%, 53.3 \%, 76.5 \%, 100 \%$ and $100 \%$, respectively. The experimental observations indicated that $\mathrm{mOmpF}$

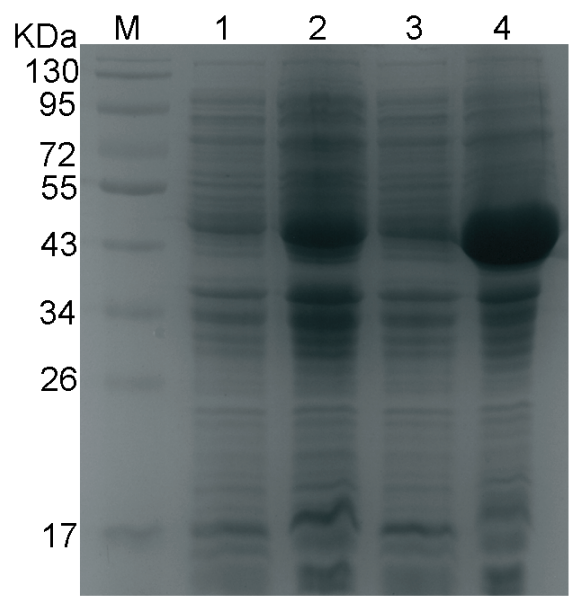

Figure 2. SDS/PAGE analysis of the expressed mOmpF.

1. BL21/pET28a in the absence of IPTG; 2 . BL21/pET28a in the presence of IPTG; 3. BL21/pET28-mOmpF in the absence of IPTG; 4. BL21/pET28-mOmpF in the presence of IPTG. was markedly mutated compared to that of the model OmpF.

Expression of mOmpF and the effects on the bacterial survival rate

SDS/PAGE implied that the mOmpF protein was successfully expressed because an approximately $40 \mathrm{kDa}$ protein band was observed as expected (see Fig. 2). No similar band was present in the control sample containing only pET28a plasmid after induction with IPTG. As expected, expression of the $\mathrm{mOmpF}$ protein significantly affected the survival of the BL21 host strains (see Table 1). The clone numbers of BL21/pET28a and BL21/pET28$\mathrm{mOmpF}$ grown in LB agar medium in the absence of 50 $\mu \mathrm{g} / \mathrm{mL}$ kanamycin were 123 and 80, respectively. However, those in the presence of $50 \mu \mathrm{g} / \mathrm{mL}$ kanamycin were 91 and 0 . Thus, the survival rates of BL21/pET28a and BL21/pET28-mOmpF were $73.98 \%$ and 0 , respectively. There was a $31 \mathrm{~mm}$ kanamycin inhibition zone with BL21/pET28-mOmpF, but none with BL21/pET28a. It could be concluded that the expression of $\mathrm{mOmpF}$ hugely affected the survival of BL21 host strains.

\section{Effects on the bacterial survival rates by different concentrations of $\mathrm{mOmpF}$}

Abundance of $\mathrm{mOmpF}$ plays critical roles in the permeation of antibiotics and the bacterial survival rate. To further explore the relationship between the $\mathrm{mOmpF}$ concentration and the bacterial survival rate, optimal induction time of $\mathrm{mOmpF}$ was studied. Samples induced for $0 \mathrm{~min}, 20 \mathrm{~min}, 40 \mathrm{~min}, 60 \mathrm{~min}$ and $120 \mathrm{~min}$ were assayed by SDS/PAGE. Protein bands for different induction times are shown in Fig. 3, which indicates that the band for $60 \mathrm{~min}$ induction is as bright as that for 120 min and brighter and wider than that for $20 \mathrm{~min}$ and 40 $\mathrm{min}$, respectively. Therefore, $60 \mathrm{~min}$ is the optimal in- 


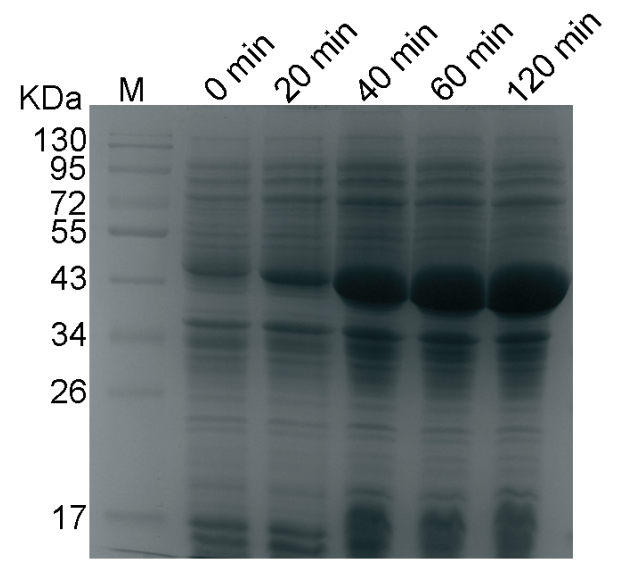

Figure 3. SDS/PAGE analysis of $\mathrm{mOmpF}$ induced by the addition of IPTG to a final concentration of $1 \mathrm{mM}$ for $0 \mathrm{~min}, 20 \mathrm{~min}, 40$ $\min , 1 \mathrm{~h}$ and $2 \mathrm{~h}$.

Table 2. Effects of mOmpF concentration on the bacterial survival rate

\begin{tabular}{llll}
\hline \multirow{2}{*}{$\begin{array}{l}\text { Induction time } \\
\text { (min) }\end{array}$} & \multicolumn{2}{l}{ Number of clones } & \\
\cline { 2 - 3 } & $\begin{array}{l}\text { LB medium } \\
\text { with kanamycin }\end{array}$ & LB medium & \\
\hline 0 & 60 & 80 & $75 \%$ \\
\hline 20 & 12 & 80 & $15 \%$ \\
\hline 40 & 7 & 81 & $8.64 \%$ \\
\hline 60 & 1 & 80 & $1.25 \%$ \\
\hline 120 & 0 & 80 & 0 \\
\hline
\end{tabular}

duction time. Comparison of the growth rate of BL21/ pET28-mOmpF with that of BL21/pET28a is shown in Fig. 4. It is clearly revealed that the growth rate of BL21/pET28-mOmpF was almost the same as that of the BL21/pET28a. All induced samples were plated on LB agar medium after serial dilution in the absence and presence of $50 \mu \mathrm{g} / \mathrm{mL}$ kanamycin. The number of clones of samples induced for $0 \mathrm{~min}, 20 \mathrm{~min}, 40 \mathrm{~min}$, $60 \mathrm{~min}$ and $120 \mathrm{~min}$ grown in the LB agar medium in

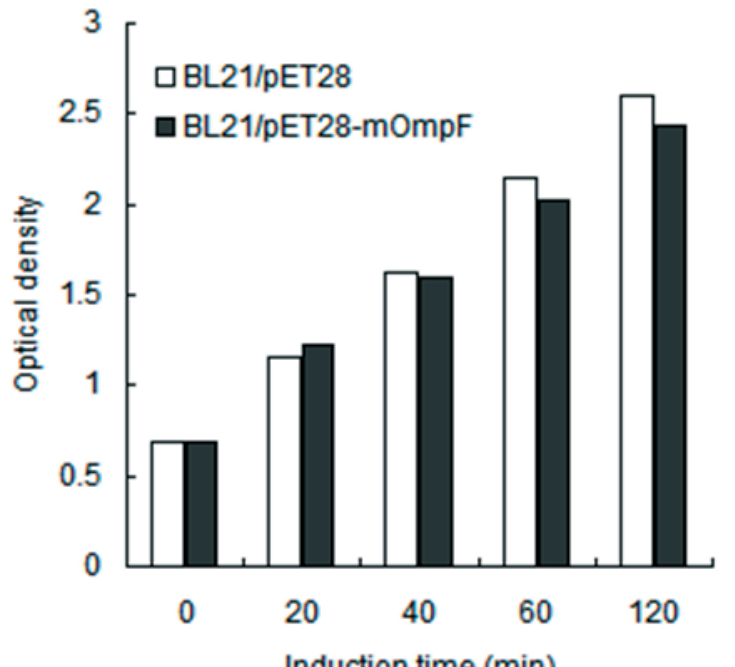

Figure 4. Growth rates of BL21/pET28-mOmpF and BL21/ pET28a.
Table 3. Effects of mOmpF concentration on the size of inhibition zone

\begin{tabular}{ll}
\hline Induction time $(\mathrm{min})$ & Inhibition zone $(\mathrm{mm})$ \\
\hline 0 & 0 \\
\hline 20 & 23 \\
\hline 40 & 25 \\
\hline 60 & 30 \\
\hline 80 & 31 \\
\hline
\end{tabular}

the absence of $50 \mu \mathrm{g} / \mathrm{mL}$ kanamycin was approximately 80 . However, growth in the presence of $50 \mu \mathrm{g} / \mathrm{mL}$ kanamycin was strongly repressed and the clone numbers were 60, 12, 7, 1 and 0 , respectively, as seen in Table 2. Consequently, the survival rate for samples induced for $0 \mathrm{~min}, 20 \mathrm{~min}, 40 \mathrm{~min}, 60 \mathrm{~min}$ and $120 \mathrm{~min}$ was $75 \%$, $15 \%, 8.64 \%, 1.25 \%$ and 0 , respectively. The abundance of $\mathrm{mOmpF}$ has remarkable effects on the size of inhibition zone, as shown in Table 3. The inhibition zone is larger for the sample induced for a longer time. It could be concluded that concentration of $\mathrm{mOmpF}$ remarkably affects the bacterial survival rate.

\section{DISCUSSION}

In the present study, we cloned a high-grade mutant OmpF from an isolated multiple antibiotic resistant E. coli strain. Sequence alignment indicated that the $m O m p F$ DNA sequence was $54.5 \%$ identical to that of the model $O m p F$ and the amino acid sequence shared only $44.6 \%$ homology. Experimental results suggested the high-grade mutant $\mathrm{mOmpF}$ still plays very important roles in decrease of bacterial survival rate.

It is known that $\mathrm{OmpF}$ is composed of 16 strand anti-parallel $\beta$-barrel with eight short turns (T1-T8) and eight long loops (L1-L8) (Cowan et al., 1992). In the present work, we revealed that the DNA sequence of the $m O m p F$ was high-grade mutated when compared to that of the model OmpF and shared only $54.5 \%$ homology, thus leading to a very low similarity in amino acid sequence. Data revealed that all of the 16 stands were mutated. All loops and short turns, except T6 and T7, were also mutated. L3 is the longest loop and contributes to a constriction of the channel where the charge distribution affects ion selectivity. Furthermore, L3 folds inside the barrel and thus affects the size of the porin (Cowan, 1995). The mOmpF in this study, with the L3 mutated at $48.5 \%$, still possessed excellent activities in antibiotic permeation, suggesting the mutations in L3 may have no significant effects on the entrance of antibiotics into cells. It has been reported that a deletion mutant of L3 $(\Delta 109-114)$ decreases the signal channel conductance by one third and drastically changes the ion selectivity (Saint et al., 1996). Lou and coworkers (1996) pointed out that a deletion mutation of the six residues $(\Delta 109$ 114) results in disorder of seven adjacent residues but does not alter the structure of the $\beta$-barrel framework (Lou et al., 1996). It is presumable that the mOmpF will change the channel conductance and ion selectivity and will not changed the configuration of the $\beta$-barrel. It has been well-characterized that L3 determines the OmpF eyelets and alteration of amino acid residues (113-124) will modify the eyelets (Chevalier et al., 1999; Simonet et al., 2000). On the other hand, as observed in Fig. 1, the amino acid residues Val104, Val105, Ala108, Leu109, 
Gly110, Tyr111, Pro116, Phe118, Thr122, Ala123, Ser125, Asp127, Phe128, Phe129, Val130 and Val133 were replaced by aLA104, Ile105, Val108, Glu109, Ala110, Ala111, Val116, Trp118, Trp122, Asn123, Thr125, Asn127, Tyr128, Met129, Thr130 and Thr133, respectively. Data in Tables 1-3 suggest that the above eleven mutated amino acids may not play important roles in L3 and the permeation of antibiotics. Furthermore, mutation at the C-terminus will not affect the function of $\mathrm{mOmpF}$ in decreasing of bacterial survival rate, as revealed in Fig. 1 and Table 1-3.

$\mathrm{OmpF}$ is the main outer membrane of E. coli allowing the diffusion of small hydrophilic molecules, such as nutrients, waste products and antibiotics, across the outer membrane (Bredin et al., 2003). Permeation through the outer membrane is the key step for an antibiotic to act on its specific intracellular target, and thus to control the permeability of the outer membrane is the first line defense to protect against antibiotics (Masi \& Pages, 2013). Additionally, it has been well understood that inhibited expression of $\mathrm{OmpF}$ will lead to bacterial antibiotic resistance (Kishii \& Takei, 2009). As shown in Fig. 3, the quantity of $\mathrm{mOmpF}$ expressed increases until $1 \mathrm{~h}$ after induction; the data together with Tables 2 and 3, suggest that high level expression of OmpF leads to a low survival rate of the bacteria and larger antibiotic inhibition zone. It has been well characterized that the outer membrane proteins play crucial roles in the permeation of antibiotics. James et al proved that concentration of outer membrane protein omp36 in Enterobacter aerogenes affects influx of $\beta$-lactams, measured by conductance (James et al., 2009). However, change of concentration of an antibiotic in the host strain should be determined for further demonstration of the $\mathrm{mOmpF}$ functions in antibiotic permeation. Additionally, much more attention should be paid to the immunogenic characterization of $\mathrm{mOmpF}$ in the next step.

\section{Acknowledgements}

This work was supported by the National Natural Science Foundation of China (No. 31100089), Scientific Research Foundation of the Education Department of Sichuan Province, China (No. 11ZB102) and the Talent Project of Sichuan University of Science \& Engineering (No. 2011RC12).

\section{REFERENCES}

Bekhit A, Fukamachi T, Saito H, Kobayashi H (2011) The role of $\mathrm{OmpC}$ and $\mathrm{OmpF}$ in acidic resistance in Escherichia coli. Biol Pharm Bull 34: 330-334.

Bredin J, Simonet V, Iyer R, Delcour AH, Pages JM (2003) Colicins, spermine and cephalosporins: a competitive interaction with the OmpF eyelet. Biochem J 376: 245-252.

Chevalier J, Pages JM, Mallea M (1999) In vivo modification of porin activity conferring antibiotic resistance to Enterobacter aerogenes. Biochem Biophys Res Commun 266: 248-251.

Cohen SP, McMurry LM, Hooper DC, Wolfson JS, Levy SB (1989) Cross-resistance to fluoroquinolones in multiple-antibiotic-resistant
(Mar) Escherichia coli selected by tetracycline or chloramphenicol: decreased drug accumulation associated with membrane changes in addition to OmpF reduction. Antimicrob Agents Chemother 33: 13181325.

Cowan SW, Garavito RM, Jansonius JN, Jenkins JA, Karlsson R, Konig N, Pai EF, Pauptit RA, Rizkallah PJ, Rosenbusch JP, Rummel G, Schirmer 'T (1995) The structure of OmpF porin in a tetragonal crystal form. Structure 3: 1041-1050.

Cowan SW, Schirmer T, Rummel G, Steiert M, Ghosh R, Pauptit RA, Jansonius JN, Rosenbusch JP (1992) Crystal structures explain functional properties of two E. coli porins. Nature 358: 727-733.

Davin-Regli A, Bolla JM, James CE, Lavigne JP, Chevalier J, Garnotel E, Molitor A, Pagès JM (2008) Membrane permeability and regulation of drug "influx and efflux" in enterobacterial pathogens. Curr Drug Targets 9: 750-759.

Dupont M, James CE, Chevalier J, Pages JM (2007) An early response to environmental stress involves regulation of OmpX and OmpF, two enterobacterial outer membrane pore-forming proteins. Antimicrob Agents Chemother 51: 3190-3198.

Fernandes F, Neves P, Gameiro P, Loura LM, Prieto M (2007) Ciprofloxacin interactions with bacterial protein OmpF: modelling of FRET from a multi-tryptophan protein trimer. Biochim Biophys Acta 1768: 2822-2830.

Ho MY, Chiou ML, Chang RC, Chen YH, Cheng CC (2010) Outer membrane protein $\mathrm{OmpF}$ involved in the transportation of polypyridyl ruthenium complexes into Escherichia coli. I Inorg Biochem 104: 614-617.

Jaffe A, Chabbert YA, Semonin O (1982) Role of porin proteins $\mathrm{OmpF}$ and $\mathrm{OmpC}$ in the permeation of beta-lactams. Antimicrob Agents Chemother 22: 942-948.

James CE, Mahendran KR, Molitor A, Bolla JM, Bessonov AN, Winterhalter M, Pagès JM (2009) How beta-lactam antibiotics enter bacteria: a dialogue with the porins. PLoS One 4: e5453.

Kefala G, Ahn C, Krupa M, Esquivies L, Maslennikov I, Kwiatkowski W, Choe S. (2010) Structures of the OmpF porin crystallized in the presence of foscholine-12. Protein Sci 19: 1117-1125.

Kishii R, Takei M (2009) Relationship between the expression of ompF and quinolone resistance in Eshcherichia coli. I Infect Chemother 15: $361-366$

Koebnik R, Locher KP, Van Gelder P (2000) Structure and function of bacterial outer membrane proteins: barrels in a nutshell. Mol Microbiol 37: 239-253.

Lou KL, Saint N, Prilipov A, Rummel G, Benson SA, Rosenbusch JP, Schirmer T (1996) Structural and functional characterization of OmpF porin mutants selected for larger pore size. I. Crystallographic analysis. I Biol Chem 271: 20669-20675.

Masi M, Pages JM (2013) Structure, function and regulation of outer membrane proteins involved in drug transport in Enterobactericeae: the OmpF/C-TolC Case. Open Microbiol J 7: 22-33.

Nikaido H (2003) Molecular basis of bacterial outer membrane permeability revisited. Microbiol Mol Biol Rev 67: 593-656.

Pages JM, James CE, Winterhalter M (2008) The porin and the permeating antibiotic: a selective diffusion barrier in Gram-negative bacteria. Nat Rev Microbiol 6: 893-903.

Saint N, Lou KL, Widmer C, Luckey M, Schirmer T, Rosenbusch JP (1996) Structural and functional characterization of $\mathrm{OmpF}$ porin mutants selected for larger pore size. II. Functional characterization. J Biol Chem 271: 20676-20680.

Schulz GE (2002) The structure of bacterial outer membrane proteins. Biochim Biophys Acta 1565: 308-317.

Simonet V, Mallea M, Pages JM (2000) Substitutions in the eyelet region distupt cefepime diffusion through the Escherichia coli $\mathrm{OmpF}$ channel. Antimicrob Agents Chemother 44: 311-315.

Yigit H, Anderson GJ, Biddle JW, Steward CD, Rasheed JK, Valera LL, McGowan JE Jr, Tenover FC (2002) Carbapenem resistance in a clinical isolate of Enterobacter aerogenes is associated with decreased expression of $\mathrm{OmpF}$ and $\mathrm{OmpC}$ porin analogs. Antimicrob Agents Chemother 46: 3817-3822.

Zhao Z, Nie X, Li Z, Zhang Z, Ding J, Xie W (2013) Amino terminus mutant OmpA from an isolated antibiotic resistant Escherichia coli still possess resistance to environmental stresses Advances in Biological Chemistry 3: 108-113. 